\title{
ON THE HILBERT FUNCTIONS OF SETS OF POINTS IN $\mathbb{P}^{1} \times \mathbb{P}^{1} \times \mathbb{P}^{1}$
}

\author{
ELENA GUARDO AND ADAM VAN TUYL
}

\begin{abstract}
Let $H_{X}$ be the trigraded Hilbert function of a set $X$ of reduced points in $\mathbb{P}^{1} \times \mathbb{P}^{1} \times \mathbb{P}^{1}$. We show how to extract some geometric information about $X$ from $H_{X}$. This note generalizes a similar result of Giuffrida, Maggioni, and Ragusa about sets of points in $\mathbb{P}^{1} \times \mathbb{P}^{1}$.
\end{abstract}

\section{InTRODUCTION}

Let $K$ be an algebraically closed field with $\operatorname{char}(K)=0$, and suppose that $X$ is a finite set of points in the projective space $\mathbb{P}^{n}$ over $K$. The Hilbert function of $X$ is the numerical function $H_{X}: \mathbb{N} \rightarrow \mathbb{N}$ defined by $H_{X}(i)=\operatorname{dim}_{K}(R / I(X))_{i}$ where $R=K\left[x_{0}, \ldots, x_{n}\right]$ and $I(X)$ is the homogeneous ideal associated to $X$. The Hilbert functions of sets of points are a well studied object, see for example [4, 5, 6, 9]. Among the questions one can ask is the question of what geometric information about the set of points, e.g., the number of collinear points in $X$, can be inferred from the function $H_{X}$. For work on problems of this type, we point the readers to [1, 2].

Giuffrida, Maggioni, and Ragusa [7] were among the first to consider the Hilbert function of a set of points $X$ in a multiprojective space $\mathbb{P}^{n_{1}} \times \cdots \times \mathbb{P}^{n_{r}}$. In this context, the associated ideal $I(X)$ is a multihomogeneous ideal in an $\mathbb{N}^{r}$-graded polynomial ring $R$. The Hilbert function of $X$ is the function $H_{X}: \mathbb{N}^{r} \rightarrow \mathbb{N}$ defined by $H_{X}(\underline{i})=\operatorname{dim}_{K}(R / I(X))_{\underline{i}}$. Unlike the singly graded case, our understanding of these functions is far from complete. Most notably, while there exists a classification for the Hilbert functions of sets of points in $\mathbb{P}^{n}$ (see [6]), no classification is known in the multigraded situation, including $\mathbb{P}^{1} \times \mathbb{P}^{1}$. Some known results can be found in [8, 7, 10].

In this note, we introduce some new results about the Hilbert functions of points $X$ in $\mathbb{P}^{1} \times \mathbb{P}^{1} \times \mathbb{P}^{1}$ (which can be scaled to $\mathbb{P}^{1} \times \cdots \times \mathbb{P}^{1}$; see Remark 3.6). In particular, in the spirit of [1, 2], we describe how geometric information about $X$ is encoded into the Hilbert function $H_{X}$. Specifically, Theorem 3.1 shows how the number of points on a "line" (see Definition 2.2) in $\mathbb{P}^{1} \times \mathbb{P}^{1} \times \mathbb{P}^{1}$ is captured. We were inspired by a similar result of Giuffrida, Maggioni, and Ragusa [7, Theorem 2.12] for points in $\mathbb{P}^{1} \times \mathbb{P}^{1}$. Our proof, however, uses a different approach.

1991 Mathematics Subject Classification. 13D40,14M99.

Key words and phrases. points, multiprojective spaces, Hilbert function. 


\section{Preliminaries}

We introduce the necessary notation and basic results concerning sets of points in $\mathbb{P}^{1} \times \mathbb{P}^{1} \times \mathbb{P}^{1}$. Throughout, we use $\succeq$ to denote the natural partial order on $\mathbb{N}^{3}$ defined by $(i, j, k) \succeq\left(i^{\prime}, j^{\prime}, k^{\prime}\right)$ if and only if $i \geq i^{\prime}, j \geq j^{\prime}$, and $k \geq k^{\prime}$. We set $R=$ $K\left[x_{0}, x_{1}, y_{0}, y_{1}, z_{0}, z_{1}\right]$ and induce a trigrading by setting $\operatorname{deg} x_{i}=(1,0,0), \operatorname{deg} y_{i}=(0,1,0)$ and $\operatorname{deg} z_{i}=(0,0,1)$ for $i=1,2$.

Suppose that

$$
P=\left[a_{0}: a_{1}\right] \times\left[b_{0}: b_{1}\right] \times\left[c_{0}: c_{1}\right] \in \mathbb{P}^{1} \times \mathbb{P}^{1} \times \mathbb{P}^{1}
$$

is a point in $\mathbb{P}^{1} \times \mathbb{P}^{1} \times \mathbb{P}^{1}$. Associated to $P$ is the trihomogeneous ideal given by

$$
I(P)=\left(a_{1} x_{0}-a_{0} x_{1}, b_{1} y_{0}-b_{0} y_{1}, c_{1} z_{0}-c_{0} z_{1}\right) .
$$

Given any finite set of distinct points $X=\left\{P_{1}, \ldots, P_{s}\right\}$ in $\mathbb{P}^{1} \times \mathbb{P}^{1} \times \mathbb{P}^{1}$, its associated ideal is the trihomogeneous ideal $I(X)=\bigcap_{i=1}^{s} I\left(P_{i}\right)$.

We will sometimes write $P$ as $P=A \times B \times C$, with $A, B, C \in \mathbb{P}^{1}$, i.e., we will use $A$ 's for the first coordinate, and so on. We shall sometimes write the generators of $I(P)$ as $I(P)=\left(L_{A}, L_{B}, L_{C}\right)$ where $L_{A}$ is the form of degree $(1,0,0), L_{B}$ is the form of degree $(0,1,0)$, and $L_{C}$ is the form of degree $(0,0,1)$.

For each $i=1,2,3$, let $\pi_{i}: \mathbb{P}^{1} \times \mathbb{P}^{1} \times \mathbb{P}^{1} \rightarrow \mathbb{P}^{1}$ denote the natural projection map. Consequently, $\pi_{1}(X)=\left\{A_{1}, \ldots, A_{t_{1}}\right\}, \pi_{2}(X)=\left\{B_{1}, \ldots, B_{t_{2}}\right\}$, and $\pi_{3}(X)=\left\{C_{1}, \ldots, C_{t_{3}}\right\}$ denote the sets of distinct first, second, and third coordinates of $X$ respectively. We let $t_{1}=\left|\pi_{1}(X)\right|, t_{2}=\left|\pi_{2}(X)\right|$, and $t_{3}=\left|\pi_{3}(X)\right|$.

Definition 2.1. The Hilbert function of $X$, denoted $H_{X}$, is the function $H_{X}: \mathbb{N}^{3} \rightarrow \mathbb{N}$ defined by $H_{X}(i, j, k)=\operatorname{dim}_{K} R_{i, j, k}-\operatorname{dim}_{K} I(X)_{i, j, k}$.

Note that $\operatorname{dim}_{K} R_{i, j, k}=(i+1)(j+1)(k+1)$ since there are $i+1$ ways to make a monomial of degree $i$ in the variables $x_{0}$ and $x_{1}, j+1$ ways to make a monomial of degree $j$ in the $y_{i} \mathrm{~s}$, and $k+1$ ways to make a monomial of degree $k$ in the $z_{i}$ s.

Our goal is to show how $H_{X}$ captures geometric information about the number of points lying on linear subvarieties of $\mathbb{P}^{1} \times \mathbb{P}^{1} \times \mathbb{P}^{1}$. We give a more precise definition.

Definition 2.2. Let $L \in R_{1,0,0}$ and $L^{\prime} \in R_{0,1,0}$. We call the variety $\mathcal{L}$ in $\mathbb{P}^{1} \times \mathbb{P}^{1} \times \mathbb{P}^{1}$ defined by the ideal $\left(L, L^{\prime}\right) \subseteq R$ a line of type $(1,1,0)$.

Remark 2.3. We will focus only on lines of type $(1,1,0)$, although similar results can be proved for lines of type $(1,0,1)$ and $(0,1,1)$, which are defined in an analogous manner.

Remark 2.4. We add a few comments about how to interpret the geometry. One can construct an embedding of $\mathbb{P}^{1} \times \mathbb{P}^{1} \times \mathbb{P}^{1}$ into a projective space; in particular, using Segre's embedding $\mathbb{T}=\mathbb{P}^{1} \times \mathbb{P}^{1} \times \mathbb{P}^{1} \hookrightarrow \mathbb{P}^{7}$ using the sheaf $\mathcal{O}_{\mathbb{T}}(1,1,1)$. We can easily check that the ideal of the image $\mathbb{T}^{\prime}$ of $\mathbb{T}$ under the embedding is:

$$
\begin{aligned}
I\left(\mathbb{T}^{\prime}\right)= & \left(u_{0} u_{7}-u_{1} u_{6}, u_{0} u_{7}-u_{2} u_{5}, u_{0} u_{7}-u_{3} u_{4}, u_{0} u_{3}-u_{1} u_{2}, u_{4} u_{7}-u_{5} u_{6},\right. \\
& \left.u_{0} u_{5}-u_{1} u_{4}, u_{2} u_{7}-u_{3} u_{6}, u_{0} u_{6}-u_{2} u_{4}, u_{1} u_{7}-u_{3} u_{5}\right)
\end{aligned}
$$


ON THE HILBERT FUNCTIONS OF SETS OF POINTS IN $\mathbb{P}^{1} \times \mathbb{P}^{1} \times \mathbb{P}^{1}$

in $K\left[u_{0}, \ldots, u_{7}\right]$. We can view $\mathbb{P}^{1} \times \mathbb{P}^{1} \times \mathbb{P}^{1}$ as ruled 3 -fold. The lines of type $(1,1,0)$, respectively $(1,0,1)$ and $(0,1,1)$, can be viewed as rulings on this surface. Our goal is to count the number of points on these rulings using the Hilbert function.

Alternatively, if we fix a degree $(1,0,0)$ form $L$, we can view this as fixing a divisor of type $(1,0,0)$. Fixing this linear equation is equivalent to fixing a point $A$ in the first copy of $\mathbb{P}^{1}$. We are then looking at points in the set

$$
S_{A}=\left\{A \times B \times C \in \mathbb{P}^{1} \times \mathbb{P}^{1} \times \mathbb{P}^{1} \mid B \times C \in \mathbb{P}^{1} \times \mathbb{P}^{1}\right\} \cong \mathbb{P}^{1} \times \mathbb{P}^{1}
$$

which is also isomorphic to the ruled quadric surface in $\mathbb{P}^{3}$. So, when we form a line of type $(1,1,0)$, we can view it as fixing a point in a $\mathbb{P}^{1}$ and a line in a $\mathbb{P}^{1} \times \mathbb{P}^{1}$, or equivalently, a ruling on the ruled quadric surface $Q$, and then we are counting the number of points on this ruling on the quadric $Q$. Note, by abuse of notation, the divisor of type $(1,0,0)$ can be called a plane of type $(1,0,0)$, and similarly for divisor of type $(0,1,0)$. So, the intersection of these two planes results in a line of type $(1,1,0)$.

We now provide a number of lemmas that shall be required for our main result.

Lemma 2.5. Let $X$ be a finite set of distinct points in $\mathbb{P}^{1} \times \mathbb{P}^{1} \times \mathbb{P}^{1}$. Fix an integer $k \geq 0$. Then

$$
H_{X}\left(t_{1}-1, t_{2}-1, k\right)=H_{X}(i, j, k) \text { for all }(i, j, k) \succeq\left(t_{1}-1, t_{2}-1, k\right) .
$$

Proof. Let $L$ be a form of degree $(1,0,0)$ that such that no point of $X$ lies on $L$. Let $L^{\prime}$ be a form of degree $(0,1,0)$ such that no point of $X$ lies on $L^{\prime}$. We observe that $L$, respectively $L^{\prime}$, is a nonzerodivsor on $R / I(X)$. Thus the multiplication map

$$
\times \bar{L}:(R / I(X))_{i, j, k} \rightarrow(R / I(X))_{i+1, j, k}
$$

is injective for all $(i, j, k)$. A similar result holds using $L^{\prime}$. Thus

$$
H_{X}\left(t_{1}-1, t_{2}-1, k\right) \leq H_{X}\left(t_{1}, t_{2}-1, k\right) \leq \cdots \leq H_{X}\left(i, t_{2}-1, k\right) \text { for } i \geq t_{1}-1
$$

and

$$
H_{X}\left(i, t_{2}-1, k\right) \leq H_{X}\left(i, t_{2}, k\right) \leq \cdots \leq H_{X}(i, j, k) \text { for } j \geq t_{2}-1 .
$$

In addition, we have the short exact sequence

$$
0 \longrightarrow R / I(X)(-1,0,0) \stackrel{\times \bar{L}}{\longrightarrow} R / I(X) \longrightarrow R /(I(X), L) \longrightarrow 0 .
$$

Now $H_{X}\left(t_{1}-1,0,0\right)=H_{X}\left(t_{1}, 0,0\right)=\left|\pi_{1}(X)\right|$ because $\bigoplus_{i \in \mathbb{N}} I(X)_{i, 0,0} \cong I\left(\pi_{1}(X)\right) \subseteq$ $K\left[x_{0}, x_{1}\right]$, i.e., the ideal of the $t_{1}$ points $\pi_{1}(X)$ in $\mathbb{P}^{1}$. So, the short exact sequence implies that $(I(X), L)_{i, 0,0}=R_{i, 0,0}$ for all $i \geq t_{1}$. But this means that the multiplication map $\times \bar{L}$ is also surjective for $(i, j, k)$ with $i \geq t_{1}-1$. Thus $H_{X}\left(t_{1}-1, t_{2}-1, k\right)=H_{X}\left(t_{1}, t_{2}-1, k\right)=$ $\cdots=H_{X}\left(i, t_{2}-1, k\right)$.

We now apply a similar argument with $L^{\prime}$ to show that $H_{X}\left(i, t_{2}-1, k\right)=H_{X}\left(i, t_{2}, k\right)=$ $\cdots=H_{X}(i, j, k)$.

Lemma 2.6. Let $\mathcal{L}$ be a line of type $(1,1,0)$, and suppose that $X$ is a finite set of distinct points in $\mathbb{P}^{1} \times \mathbb{P}^{1} \times \mathbb{P}^{1}$ such that $X \subseteq \mathcal{L}$. If $|X|=s$, then

$$
H_{X}(i, j, k)=\min \{k+1, s\} \text { for all }(i, j, k) \in \mathbb{N}^{3} \text {. }
$$


Proof. Because $X \subseteq \mathcal{L}$, the set $X$ has the form

$$
X=\left\{A \times B \times C_{1}, A \times B \times C_{2}, \ldots, A \times B \times C_{s}\right\}
$$

for some $A, B, C_{i} \in \mathbb{P}^{1}$. After a change of coordinates, we can assume that $A=[1: 0]$ and $B=[1: 0]$. Thus

$$
I(X)=\bigcap_{i=1}^{s}\left(x_{1}, y_{1}, L_{C_{i}}\right)=\left(x_{1}, y_{1}, L_{C_{1}} L_{C_{2}} \cdots L_{C_{s}}\right) .
$$

If we set $L=L_{C_{1}} \cdots L_{C_{s}}$, then $R / I(X) \cong K\left[x_{0}, y_{0}, z_{0}, z_{1}\right] /(L)$; here, we are viewing $L$ as an element of $K\left[x_{0}, y_{0}, z_{0}, z_{1}\right]$. If $S=K\left[x_{0}, y_{0}, z_{0}, z_{1}\right]$ is the $\mathbb{N}^{3}$-graded ring with $\operatorname{deg} x_{0}=(1,0,0), \operatorname{deg} y_{0}=(0,1,0)$ and $\operatorname{deg} z_{i}=(0,0,1)$, then the result follows by using the short exact sequence

$$
0 \longrightarrow S(0,0,-s) \stackrel{\times L}{\longrightarrow} S \longrightarrow S /(L) \longrightarrow 0
$$

to compute $H_{X}$ and the fact that $\operatorname{dim}_{K} S_{i, j, k}=k+1$ for all $(i, j) \in \mathbb{N}^{2}$.

Lemma 2.7. Let $X$ be a finite set of distinct points in $\mathbb{P}^{1} \times \mathbb{P}^{1} \times \mathbb{P}^{1}$, and suppose that $\mathcal{L}$ is a line of type $(1,1,0)$ that intersects $X$, but $X \nsubseteq \mathcal{L}$. Set $X_{2}=X \cap \mathcal{L}$ and $X_{1}=X \backslash X_{2}$. Then

$$
R_{t_{1}-1, t_{2}-1,0}=\left(I\left(X_{1}\right)+I\left(X_{2}\right)\right)_{t_{1}-1, t_{2}-1,0}
$$

Consequently,

$$
H_{R /\left(I\left(X_{1}\right)+I\left(X_{2}\right)\right)}\left(t_{1}-1, t_{2}-1, k\right)=0 \text { for all } k \geq 0 \text {. }
$$

Proof. The second statement follows from the first since $\left(I\left(X_{1}\right)+I\left(X_{2}\right)\right)_{t_{1}-1, t_{2}-1, k}=$ $R_{t_{1}-1, t_{2}-1, k}$ for all $k \geq 0$ if the first statement holds.

After a change of coordinates, we can assume that $\mathcal{L}$ is the line defined by $x_{1}$ and $y_{1}$, i.e., every point on $X_{2}$ has the form $[1: 0] \times[1: 0] \times C$ for some $C \in \mathbb{P}^{1}$. As in the proof of Lemma 2.6, we can take $I\left(X_{2}\right)=\left(x_{1}, y_{1}, L\right)$ where $L$ is some homogeneous polynomial only in the variables $z_{0}$ and $z_{1}$. Now for any $(i, j) \in \mathbb{N}^{2}, I\left(X_{2}\right)_{i, j, 0}$ contains all the monomials of degree $(i, j, 0)$ except $x_{0}^{i} y_{0}^{j}$.

Now $\pi_{1}(X)=\left\{A_{1}, \ldots, A_{t_{1}-1},[1: 0]\right\}$ and $\pi_{2}(X)=\left\{B_{1}, \ldots, B_{t_{2}-1},[1: 0]\right\}$. Note that because $X \nsubseteq \mathcal{L}$, either $t_{1} \geq 2$ or $t_{2} \geq 2$. Let $L_{A_{i}}$ be the form of degree $(1,0,0)$ that vanishes at points of the form $A_{i} \times B \times C$ where $B, C \in \mathbb{P}^{1}$ and let $L_{B_{j}}$ be the form of degree $(0,1,0)$ that vanishes at all points of the form $A \times B_{j} \times C$ with $A, C \in \mathbb{P}^{1}$. Then $H=L_{A_{1}} \cdots L_{A_{t_{1}-1}} L_{B_{1}} \ldots, L_{B_{t_{2}-1}}$ is a degree $\left(t_{1}-1, t_{2}-1,0\right)$ form that vanishes at all the points of $X_{1}$ with $t_{1}-1>0$ or $t_{2}-1>0$, that is, $\left(t_{1}-1, t_{2}-1,0\right) \neq(0,0,0)$. Thus $H \in I\left(X_{1}\right)_{t_{1}-1, t_{2}-1,0}$ and $H$ is not a constant. Furthermore, since $H$ does not vanish at any of the points in $X_{2}$, neither $x_{1}$ nor $y_{1}$ divides $H$. So $H$ must have the form $H=c x_{0}^{t_{1}-1} y_{0}^{t_{2}-1}+H^{\prime}$ where $c \neq 0$.

Since $H \in I\left(X_{1}\right)_{t_{1}-1, t_{2}-1,0}$ and since all the monomials of degree $\left(t_{1}-1, t_{2}-1,0\right)$ except $x_{0}^{t_{1}-1} y_{0}^{t_{2}-1}$ belong to $I\left(X_{2}\right)_{t_{1}-1, t_{2}-1,0}$, we must have all the monomials of degree $\left(t_{1}-1, t_{2}-\right.$ $1,0)$ belonging to $\left(I\left(X_{1}\right)+I\left(X_{2}\right)\right)_{t_{1}-1, t_{2}-1,0}$, from which our conclusion follows. 


\section{Main Result}

In this section we prove our main result which explains how to extract geometric information about $X$ from $H_{X}$. In particular, we are able to determine information about the number of points on lines of type $(1,1,0)$.

We make use of the following notation. If $X$ is a finite set of distinct points in $\mathbb{P}^{1} \times$ $\mathbb{P}^{1} \times \mathbb{P}^{1}$, for each integer $i \geq 1$, let

$r_{i}(X):=$ the number of lines of type $(1,1,0)$ that contain exactly $i$ points of $X$.

Because $X$ is finite, $r_{i}(X)=0$ for all but a finite number of $i$. Given $H_{X}$, we set

$$
d_{i, j, k}:=H_{X}(i, j, k)-H_{X}(i, j, k-1) \text { for all }(i, j, k) \in \mathbb{N}^{3}
$$

where $H_{X}(a, b, c)=0$ if $(a, b, c) \nsucceq(0,0,0)$. We shall be interested in the sequence $\left\{d_{t_{1}-1, t_{2}-1, k}-d_{t_{1}-1, t_{2}-1, k+1}\right\}_{k \in \mathbb{N}}$. It is not obvious that this is a sequence of nonnegative numbers. However, even though one can prove this directly from the properties of the Hilbert function, this fact will be an immediate consequence of our main theorem, stated below, which shows how geometric information is encoded into the sequence.

Theorem 3.1. Let $X$ be a finite set of distinct points in $\mathbb{P}^{1} \times \mathbb{P}^{1} \times \mathbb{P}^{1}$. Let $t_{1}=\left|\pi_{1}(X)\right|$ and $t_{2}=\left|\pi_{2}(X)\right|$. For every $k \geq 0$,

$$
\begin{aligned}
r_{k+1}(X) & =d_{t_{1}-1, t_{2}-1, k}-d_{t_{1}-1, t_{2}-1, k+1} \\
& =2 H_{X}\left(t_{1}-1, t_{2}-1, k\right)-H_{X}\left(t_{1}-1, t_{2}-1, k-1\right)-H_{X}\left(t_{1}-1, t_{2}-1, k+1\right) .
\end{aligned}
$$

Remark 3.2. As we see in the above theorem, $r_{k+1}(X)$ can be computed directly from the Hilbert function $H_{X}$ or through the $d_{i, j, k} \mathrm{~s}$. We have presented the formula for $r_{k+1}(X)$ using the $d_{i, j, k}$ s because it is reminiscent of the formulas found in [7, Theorem 2.12] for points in $\mathbb{P}^{1} \times \mathbb{P}^{1}$ which inspired this result.

Let us illustrate this result before turning to its proof.

Example 3.3. We show how to go from the Hilbert function to information about the set of points. Suppose that we are given the following trigraded Hilbert function for a set of points $X$. Below, $H_{X}$ is written as a collection of infinite matrices, where the initial row and column are indexed with 0 as opposed to 1 . You should view these matrices as the "layers" of the box matrix that make up $H_{X}$.

$$
H_{X}(i, j, 0)=\left[\begin{array}{ccccc}
1 & 2 & 3 & 3 & \cdots \\
2 & 4 & 5 & 5 & \cdots \\
3 & 5 & 6 & 6 & \cdots \\
3 & 5 & 6 & 6 & \cdots \\
\vdots & \vdots & \vdots & \vdots & \ddots
\end{array}\right] \quad H_{X}(i, j, 1)=\left[\begin{array}{ccccc}
2 & 4 & 5 & 5 & \cdots \\
4 & 7 & 8 & 8 & \cdots \\
5 & 8 & 9 & 9 & \ldots \\
5 & 8 & 9 & 9 & \cdots \\
\vdots & \vdots & \vdots & \vdots & \ddots
\end{array}\right] \quad H_{X}(i, j, 2)=\left[\begin{array}{ccccc}
3 & 6 & 7 & 7 & \cdots \\
5 & 9 & 10 & 10 & \cdots \\
6 & 10 & 11 & 11 & \cdots \\
6 & 10 & 11 & 11 & \cdots \\
\vdots & \vdots & \vdots & \vdots & \ddots
\end{array}\right]
$$

and $H_{X}(i, j, k)=H_{X}(i, j, 2)$ for all $k \geq 2$.

From this Hilbert function, we can see that $t_{1}=t_{2}=t_{3}=3$. We can use Theorem 3.1 to determine the number of points on each line of type $(1,1,0)$. We need the values of $H_{X}(2,2, l)$ for all $l \in \mathbb{N}$. From $H_{X}$ above, we have

$$
H_{X}(2,2,0)=6, H_{X}(2,2,1)=9 \text {, and } H_{X}(2,2, l)=11 \text { for all } l \geq 2 .
$$


So, $d_{2,2,0}=6, d_{2,2,1}=3, d_{2,2,2}=2$, and $d_{2,2, l}=0$ otherwise. By Theorem $[3.1$, we then have $d_{2,2,0}-d_{2,2,1}=3$ lines of type $(1,1,0)$ that contain exactly one point of $X, d_{2,2,1}-d_{2,2,2}=1$ lines of type $(1,1,0)$ that contain exactly two points of $X$, and $d_{2,2,2}-d_{2,2,3}=2$ lines of type $(1,1,0)$ that contain exactly three points of $X$.

Indeed, $H_{X}$ is the Hilbert function of the set of points $X$ constructed as below. Let $A_{i}:=[1: i] \in \mathbb{P}^{1}, B_{i}:=[1: i] \in \mathbb{P}^{1}$ and $C_{i}:=[1: i] \in \mathbb{P}^{1}$. Then $X$ is the following scheme

$$
X:=\left\{P_{111}, P_{112}, P_{113}, P_{121}, P_{122}, P_{123}, P_{212}, P_{211}, P_{311}, P_{221}, P_{131}\right\}
$$

where $P_{i j k}=A_{i} \times B_{j} \times C_{k}$. If $L_{A_{i}}$ denotes the $(1,0,0)$ form that vanishes at all points of $\mathbb{P}^{1} \times \mathbb{P}^{1} \times \mathbb{P}^{1}$ whose first coordinate is $A_{i}$, and similarly, if $L_{B_{j}}$ denotes the $(0,1,0)$ form that vanishes at all points whose second coordinate is $B_{j}$, then $\left(L_{A_{1}}, L_{B_{1}}\right)$ and $\left(L_{A_{1}}, L_{B_{2}}\right)$ are the two lines of type $(1,1,0)$ that contain exactly three points of $X$.

Theorem 3.1 will follow from the next result.

Theorem 3.4. Let $X$ be a finite set of distinct points in $\mathbb{P}^{1} \times \mathbb{P}^{1} \times \mathbb{P}^{1}$. Let $t_{1}=\left|\pi_{1}(X)\right|$ and $t_{2}=\left|\pi_{2}(X)\right|$. Then for all $k \geq 0$,

$$
H_{X}\left(t_{1}-1, t_{2}-1, k\right)=\sum_{m=1}^{k+1}\left(\sum_{n=k+1}^{\infty} r_{n}(X)\right) .
$$

Proof. We do induction on the number of lines of type $(1,1,0)$ that intersect with $X$. For the base case, suppose that there is only one line $\mathcal{L}$ of type $(1,1,0)$ that intersects $X$. In other words, $X$ is contained on $\mathcal{L}$, whence $t_{1}=t_{2}=1$. If $|X|=s$, then $r_{s}(X)=1$ and $r_{s^{\prime}}(X)=0$ for all $s^{\prime} \neq s$. By Lemma 2.6,

$$
H_{X}(0,0, k)=\min \{k+1, s\} \text { for all } k \geq 0 .
$$

On the other hand, note that $\sum_{n=k+1}^{\infty} r_{n}(X)=1$ if $k+1 \leq s$ and zero otherwise. This implies that

$$
\sum_{m=1}^{k+1}\left(\sum_{n=k+1}^{\infty} r_{n}(X)\right)=\min \{k+1, s\}
$$

So the formulas agree.

For the induction step, suppose that there are $t>1$ lines $\mathcal{L}_{1}, \ldots, \mathcal{L}_{t}$ of type $(1,1,0)$ that intersect $X$. Set $X_{2}=X \cap \mathcal{L}_{t}$ and $X_{1}=X \backslash X_{2}$. We now consider the following short exact sequence

$$
0 \longrightarrow R /\left(I\left(X_{1}\right) \cap I\left(X_{2}\right)\right) \longrightarrow R / I\left(X_{1}\right) \oplus R / I\left(X_{2}\right) \longrightarrow R /\left(I\left(X_{1}\right)+I\left(X_{2}\right)\right) \longrightarrow 0 .
$$

From this short exact sequence we have

(3.1) $H_{X}\left(t_{1}-1, t_{2}-1, k\right)=H_{X_{1}}\left(t_{1}-1, t_{2}-1, k\right)+H_{X_{2}}\left(t_{1}-1, t_{2}-1, k\right)-H_{R /\left(I\left(X_{1}\right)+I\left(X_{2}\right)\right)}\left(t_{1}-1, t_{2}-1, k\right)$ for all $k \geq 0$.

Since there are only $t-1$ lines of type $(1,1,0)$ that intersect with $X_{1}$ and only one line of type $(1,1,0)$ that intersects with $X_{2}$, the induction hypothesis gives

$$
H_{X_{1}}\left(t_{1}\left(X_{1}\right)-1, t_{2}\left(X_{1}\right)-1, k\right)=\sum_{m=1}^{k+1}\left(\sum_{n=k+1}^{\infty} r_{n}\left(X_{1}\right)\right)
$$


and

$$
H_{X_{2}}\left(t_{1}\left(X_{2}\right)-1, t_{2}\left(X_{2}\right)-1, k\right)=\sum_{m=1}^{k+1}\left(\sum_{n=k+1}^{\infty} r_{n}\left(X_{2}\right)\right)
$$

where $t_{i}\left(X_{j}\right)=\left|\pi_{i}\left(X_{j}\right)\right|$ for $i=1,2$ and $j=1,2$. Because $t_{1} \geq t_{1}\left(X_{1}\right), t_{1}\left(X_{2}\right)$ and $t_{2} \geq t_{2}\left(X_{1}\right), t_{2}\left(X_{2}\right)$, Lemma 2.5 then implies that

$$
\begin{aligned}
& H_{X_{1}}\left(t_{1}-1, t_{2}-1, k\right)=\sum_{m=1}^{k+1}\left(\sum_{n=k+1}^{\infty} r_{n}\left(X_{1}\right)\right) \text { and } \\
& H_{X_{2}}\left(t_{1}-1, t_{2}-1, k\right)=\sum_{m=1}^{k+1}\left(\sum_{n=k+1}^{\infty} r_{n}\left(X_{2}\right)\right) .
\end{aligned}
$$

By Lemma 2.7. $H_{R /\left(I\left(X_{1}\right)+I\left(X_{2}\right)\right)}\left(t_{1}-1, t_{2}-1, k\right)=0$ for all $k \geq 0$. Furthermore, $r_{n}(X)=r_{n}\left(X_{1}\right)+r_{n}\left(X_{2}\right)$ for all $n \geq 1$. Thus, (3.1) gives the desired formula:

$$
\begin{aligned}
H_{X}\left(t_{1}-1, t_{2}-1, k\right) & =\sum_{m=1}^{k+1}\left(\sum_{n=k+1}^{\infty} r_{n}\left(X_{1}\right)\right)+\sum_{m=1}^{k+1}\left(\sum_{n=k+1}^{\infty} r_{n}\left(X_{2}\right)\right) \\
& =\sum_{m=1}^{k+1}\left(\sum_{n=k+1}^{\infty}\left(r_{n}\left(X_{1}\right)+r_{n}\left(X_{2}\right)\right)\right)=\sum_{m=1}^{k+1}\left(\sum_{n=k+1}^{\infty} r_{n}(X)\right) .
\end{aligned}
$$

Example 3.5. In this example, we show how Theorem 3.4 can be used to determine values of $H_{X}$ directly from information about the set of points $X$.

Let $A_{i}:=[1: i] \in \mathbb{P}^{1}, B_{i}:=[1: i] \in \mathbb{P}^{1}$ and $C_{i}:=[1: i] \in \mathbb{P}^{1}$, and suppose that $X$ is the following scheme

$$
X:=\left\{P_{111}, P_{121}, P_{122}, P_{132}, P_{133}, P_{211}, P_{221}, P_{222}, P_{232}, P_{233}\right\}
$$

where $P_{i j k}=A_{i} \times B_{j} \times C_{k}$. We can visualize this set of points as follows: 


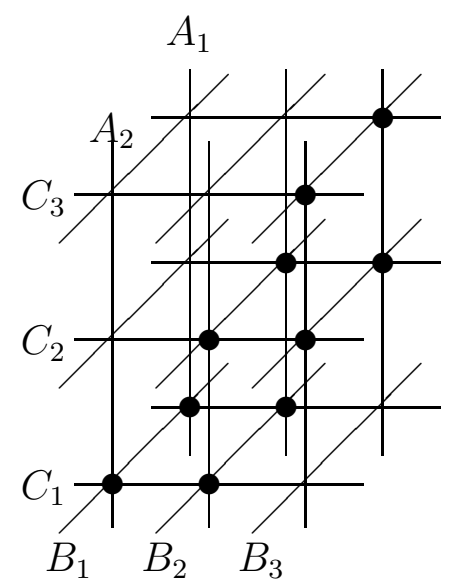

Note that in the above picture, the vertical lines represent the lines of type $(1,1,0)$.

From $X$, we can compute $r_{i}(X)$ for all $i$. First, let $L_{A_{i}}$ denote the $(1,0,0)$ form that vanishes at all points of $\mathbb{P}^{1} \times \mathbb{P}^{1} \times \mathbb{P}^{1}$ whose first coordinate is $A_{i}$, and similarly, let $L_{B_{j}}$ denote the $(0,1,0)$ form that vanishes at all points whose second coordinate is $B_{j}$. Then the line of type $(1,1,0)$ given by $\left(L_{A_{1}}, L_{B_{1}}\right)$ contains one point of $X$, the line of type $(1,1,0)$ given by $\left(L_{A_{1}}, L_{B_{2}}\right)$ contains two points of $X$, the line of type $(1,1,0)$ given by $\left(L_{A_{1}}, L_{B_{3}}\right)$ contains two points, the line of type $(1,1,0)$ given by $\left(L_{A_{2}}, L_{B_{1}}\right)$ contains one point, the line of type $(1,1,0)$ given by $\left(L_{A_{2}}, L_{B_{2}}\right)$ contains two points, and the line of type $(1,1,0)$ given by $\left(L_{A_{2}}, L_{B_{3}}\right)$ contains two points.

From this data, and from $X$, we have $t_{1}=2$ and $t_{2}=3$, and $r_{1}(X)=2, r_{2}(X)=4$, and $r_{i}(X)=0$ for $i \geq 3$. So,

$$
\begin{aligned}
& H_{X}(1,2,0)=r_{1}(X)+r_{2}(X)=6 \\
& H_{X}(1,2,1)=r_{1}(X)+r_{2}(X)+r_{2}(X)=10 \\
& H_{X}(1,2,2)=r_{1}(X)+r_{2}(X)+r_{2}(X)=10=H_{X}(1,2, l) \text { for } l \geq 2 .
\end{aligned}
$$

Note that by Lemma 2.5, we have actually computed an infinite number of values of $H_{X}$. For example, for all $(i, j, 2) \succeq(1,2,2)$, we have $H_{X}(i, j, 2)=H_{X}(1,2,2)=10$.

As a reminder, a similar result to Theorem 3.4 also holds for the number of points on a line of type $(1,0,1)$ and $(0,1,1)$. Since $t_{3}=3$, we can also compute the values of $H_{X}\left(t_{1}-1, k, t_{3}-1\right)$ and $H_{X}\left(k, t_{2}-1, t_{3}-1\right)$ for all $k \geq 0$. We omit the details.

Proof of Theorem 3.1. For any $k \geq 0$,

$$
\begin{aligned}
d_{t_{1}-1, t_{2}-1, k} & =H_{X}\left(t_{1}-1, t_{2}-1, k\right)-H_{X}\left(t_{1}-1, t_{2}-1, k-1\right) \\
& =\sum_{m=1}^{k+1}\left(\sum_{n=k+1}^{\infty} r_{n}(X)\right)-\sum_{m=1}^{k}\left(\sum_{n=k}^{\infty} r_{n}(X)\right)=\sum_{n=k+1}^{\infty} r_{n}(X) .
\end{aligned}
$$


Thus

$$
d_{t_{1}-1, t_{2}-1, k}-d_{t_{1}-1, t_{2}-1, k+1}=\sum_{n=k+1}^{\infty} r_{n}(X)-\sum_{n=k+2}^{\infty} r_{n}(X)=r_{k+1}(X)
$$

Remark 3.6. We point out that although Theorem 3.1 is only stated for points in $\mathbb{P}^{1} \times \mathbb{P}^{1} \times \mathbb{P}^{1}$, one can adapt the proofs to scale this result to $\mathbb{P}^{1} \times \cdots \times \mathbb{P}^{1}$ ( $r$ copies $)$. In particular, one defines a line of type $(\underbrace{1, \ldots, 1}_{r}, 0)$ in $\mathbb{P}^{1} \times \cdots \times \mathbb{P}^{1}$ as the variety defined by linear forms of degree $e_{1}, \ldots, e_{r-1}$ where $e_{i}$ is the standard basis vector of $\mathbb{N}^{r}$. If $t_{i}=\left|\pi_{i}(X)\right|$ is the number of distinct $i$-th coordinates that appear in $X$, then the number of points on a lines of type $(1, \ldots, 1,0)$ is encoded into the sequence

$$
\left\{H_{X}\left(t_{1}-1, \ldots, t_{r-1}-1, k\right)\right\}_{k \in \mathbb{N}} .
$$

Our result can also be seen as a generalization of [7, Theorem 2.12] which determined the geometric information encoded into the sequence $\left\{H_{X}\left(t_{1}-1, k\right)\right\}_{k \in \mathbb{N}}$ when $X$ is a set of points in $\mathbb{P}^{1} \times \mathbb{P}^{1}$ with $t_{1}=|X|$.

Acknowledgments. The computer algebra system CoCoA [3] inspired many of the results of this paper. The second author acknowledges the support of NSERC. We thank the referee for their careful reading of the paper and their invaluable suggestions.

\section{REFERENCES}

[1] A. Bigatti, A.V. Geramita, J. Migliore. Geometric consequences of extremal behavior in a theorem of Macaulay. Trans. Amer. Math. Soc. 346 (1994) 203-235.

[2] L. Chiantini, J. Migliore. Almost maximal growth of the Hilbert function. Preprint (2014) arXiv:1403.1409

[3] CoCoATeam. CoCoA: A system for doing Computations in Commutative Algebra. Available at http://cocoa.dima.unige.it

[4] A.V. Geramita, D. Gregory, L.G. Roberts. Monomial ideals and points in projective space. J. Pure Appl. Algebra 40 (1986) 33-62.

[5] A.V. Geramita, T. Harima, Y.S. Shin. An alternative to the Hilbert function for the ideal of a finite set of points in $\mathbb{P}^{n}$. Illinois J. Math. 45 (2001) 1-23.

[6] A.V. Geramita, P. Maroscia, L.G. Roberts. The Hilbert function of a reduced k-algebra. J. London Math. Soc. 28 (1983) 443-452.

[7] S. Giuffrida, R. Maggioni, A. Ragusa. On the postulation of 0-dimensional subschemes on a smooth quadric. Pacific J. Math. 155 (1992) 251-282.

[8] E. Guardo. Fat point schemes on a smooth quadric. J. Pure Appl. Algebra 162 (2001) 183-208.

[9] R. Maggioni, A. Ragusa. The Hilbert function of generic plane sections of curves of $\mathbb{P}^{3}$. Invent. Math. 91 (1988) 253-258.

[10] A. Van Tuyl. The border of the Hilbert function of a set of points in $\mathbb{P}^{n_{1}} \times \cdots \times \mathbb{P}^{n_{k}}$. J. Pure Appl. Algebra 176 (2002) 223-247. 
ON THE HILBERT FUNCTIONS OF SETS OF POINTS IN $\mathbb{P}^{1} \times \mathbb{P}^{1} \times \mathbb{P}^{1}$

Dipartimento di Matematica e Informatica, Viale A. Doria, 6 - 95100 - Catania, Italy

E-mail address: guardo@dmi.unict.it

URL: http://www.dmi.unict.it/ guardo/

Department of Mathematical Sciences, Lakehead University, Thunder Bay, ON P7B 5E1, CANADA

E-mail address: avantuyl@lakeheadu.ca

URL: http://flash.lakeheadu.ca/ avantuyl/ 